\title{
DIELECTRIC STUDIES OF CERIUM DOPED BARIUM TITANATE AT DIFFERENT FREQUENCIES
}

\author{
S. N. RAHMAN, N. KHATUN, MD. TOFAZZAL HOSSAIN \\ Industrial Physics Division, Bangladesh Council of Scientific and Industrial Research \\ (BCSIR), Dhaka.
}

AND

\author{
A.H. KHAN \\ Jahangirnagar University Savar, Dhaka.
}

\begin{abstract}
Cerium doped barium titanate is prepared by conventional ceramic method. The dielectric studies of $\mathrm{Ba}_{1-\mathrm{x}} \mathrm{Ce}_{\mathrm{x}} \mathrm{TiO}_{3}$ have been accomplished at room temperature over a frequency range 75 $\mathrm{KHz}$ to $30 \mathrm{MHz}$ for $\mathrm{x}=0.1$ to 0.4 with pure $\mathrm{BaTiO}_{3}$. The measurements of the real and imaginary part of dielectric constants show strong frequency dependence. The relaxation phenomena that take place cane be attributable to the damping of dipole oscillator due to the application of external field. In the present experiment relaxation occurs at the frequency of about $\sim 1 \mathrm{MHZ}$.
\end{abstract}

\section{INTRODUCTION}

The uses of $\mathrm{BaTiO}_{3}$ in electronic industries are increasing day by day. The utility, variety and versatility of these materials render these materials very useful and indispensable. There is thus a growing need of developing, controlling and diversification of these materials. The most promising properties of $\mathrm{BaTiO}_{3}$ are the ferroelectric properties. The ferroelectric properties of $\mathrm{BaTiO}_{3}$ strongly depend on microstructure, additives and various compositions and also on various manufacturing processes. The effect of rare earth additives on the microstructure and dielectric properties of $\mathrm{BaTiO}_{3}$ has been studied by many investigators ${ }^{(1-3)}$. Mazdysni and Brown ${ }^{(4)}$ found that a small additive of $\mathrm{La}_{2} \mathrm{O}_{3}$ enormously increases both dielectric constant and the dissipation factor whereas the Curie point is decreased. Cerium doped $\mathrm{BaTiO}_{3}$ has been extensively studied because of its high endurance under dc field stress, grain growth inhibition and effective Curie temperature shift ${ }^{(5-13)}$. Although a large number of literatures on temperature dependant characteristics are published, there are only a few work on frequency dependent dielectric constant and conductivity of cerium doped $\mathrm{BaTiO}_{3}$. Since these materials are used in electronic circuits, the studies of dielectric properties of these materials are essential for knowing the overall characteristics, such as structure, grain size, grain boundary etc. The dielectric response of barium based mixed B-sites cation perovskites is known to depend on the long range chemical ordering ${ }^{(14)}$. The magnitude of both the dielectric constant $\epsilon^{\prime}$ and the dissipation factor tan $\delta$ have been reported ${ }^{(15)}$ to decrease with the increase of frequency. The objective of this paper is to investigate the frequency characteristics of some intrinsic properties of cerium doped barium titanate 
with different compositions and to compare them with the frequency characteristics of pure barium titanate.

\section{MATERIALS AND METHODS}

2.1 Sample preparation : Cerium doped $\mathrm{BaTiO}_{3}$ used in the present experiment is prepared by conventional ceramic method of mixing $\mathrm{BaO}, \mathrm{TiO}_{2}$ and $\mathrm{CeO}_{2}$ by solid state reaction technique. All chemicals were procured from the local market. The purity of the chemicals are of analytical grade as stated by manufacturer (BDH). Specimens are prepared by weighing $\mathrm{BaO}, \mathrm{TiO}_{2}$ and $\mathrm{CeO}_{2}$ using the general formula $\mathrm{Ba}_{1-\mathrm{x}} \mathrm{Ce}_{\mathrm{x}} \mathrm{TiO}_{3}$, where $\mathrm{x}=0.1$ to 0.4 . The chemicals are grounded and 400 mesh sieve is used to get fine particle. In order to make them moisture free, the chemicals are dried in an oven at $120^{\circ} \mathrm{C}$ for two hours. Then the mixture is presintered at $900^{\circ} \mathrm{C}$ for three hours and is then allowed to cool to room temperature. The required quantity of presintered powder is taken to separate in a small glass vessel and is mixed well with some drops of water for uniform binding. The presintered powder is grounded and is mixed with distilled water and is then pressed into disks of about $\sim 6 \mathrm{~mm}$. diameter and approximately of $2 \mathrm{~mm}$. thickness. The pressed samples are sintered at $1250^{\circ} \mathrm{C}$ for three hours in a furnace.

2.2 Measurements : For the dielectric characterization, the sintered pellets are polished and silver paste is used on both sides of the pellets for good electrical contact. The frequency dependence dielectric constant is measured by precision LCR meter, Agilent 4285A (75 KHz to 30MHz) installed in BCSIR Laboratories, Dhaka. The dielectric constant and conductivity are calculated from measured capacitance and conductance using LCR meter over the frequency range $75 \mathrm{KHz}$ to $30 \mathrm{MHz}$ across the specimen under test. The measurements are taken at room temperature. X-Ray diffraction (XRD) measurements are carried out at room temperature on crushed compact disk using Philip X-Pert diffractometer and $\mathrm{CuK}_{\alpha}$ radiation. The lattice constants are calculated by the extrapolation method using the function:

$$
\frac{1}{2}\left(\frac{\cos ^{2} \theta}{\sin \theta}+\frac{\cos ^{2} \theta}{\theta}\right)
$$

\section{RESULTS AND DISCUSSIONS}

3.1 X-Ray diffraction studies: X-ray diffraction patterns confirm that the synthesized $\mathrm{BaTiO}_{3}$ is in crystalline phase. There is no significant separation between $\mathrm{TiO}_{2}$ and $\mathrm{BaO}$. The lattice parameter ratio c/a measurements for different Ce additives are shown in Table 1. The concentration of $\mathrm{CeO}_{2}$ vs. c/a ratio is shown in Fig. 1. It is evident that for pure $\mathrm{BaTiO}_{3}$, c/a ratio is found to be 1.008, which confirms perovskite structure. For $\mathrm{x}=$ $0.1 \mathrm{c} / \mathrm{a}$ ratio decreases but as $\mathrm{x}$ increases up to 0.4 , c/a ratio continuously increases up to 1.0127. It is observed that c/a ratio is highly sensitive to the doping element. Thus increasing $\mathrm{Ce}$ concentration in $\mathrm{BaTiO}_{3}$, lattice constant shows a tendency of transformation in the crystal structure from tetragonal to hexagonal at room temperature. Interestingly c/a ratio does not only depend on concentration of dopant element (Ce in this case) but also depends on partilce size of the prepared specimen. 


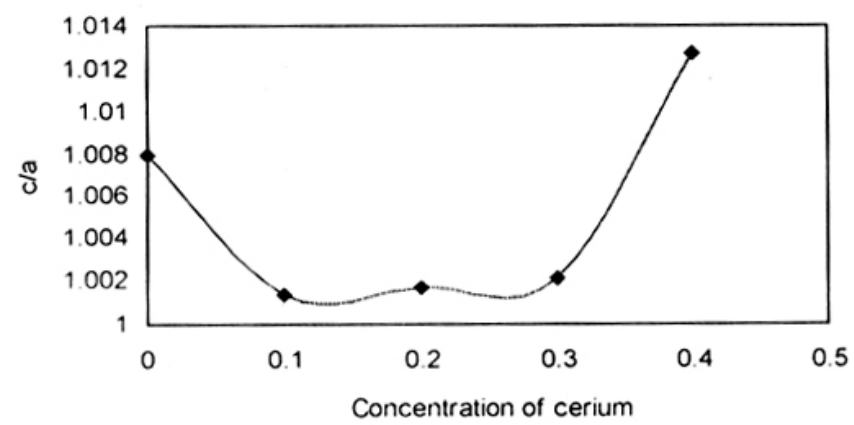

Fig. 1. c/a vs. Concentration of $\mathrm{CeO}_{2}$.

\subsection{Dielectric Properties}

Fig. 2 shows that the dielectric constant decreases with the increase of frequency. At very low frequency dipoles follow the field as the frequency increases dipoles begin to lag behind the field and $\varepsilon^{\prime}$ slightly decreases. When frequency attains a certain particular value, characteristics of the material, the dielectric constant drops (relaxation process). This is the normal behavior of dielectric material. So the dielectric constant decreases at high frequency and vice versa. Similar behavior is also observed in the present experiment on Ce-doped $\mathrm{BaTiO}_{3}$. Molokhia et al ${ }^{(16)}$ observed similar behavior in the case of ytterbium doped $\mathrm{BaTiO}_{3}$. Electrical conductivity effects give rise to higher values of $\varepsilon^{\prime \prime}$ as shown in Fig. 3., increasing with the decreasing frequency. The relation between $\sigma_{\text {ac }}$ and $\varepsilon^{\prime \prime}$ is given by $\sigma_{\text {ac }}=\varepsilon_{0} \omega \varepsilon^{\prime \prime}$. Here $\sigma_{\text {ac }}$ is the ac conductivity, $\omega=2 \pi \mathrm{f}$ (f is the measuring frequency) and $\varepsilon_{0}$ is the free space permittivity. Fig. 4. shows the plot of frequency dependence of ac conductivity of cerium doped barium titanate with the pure

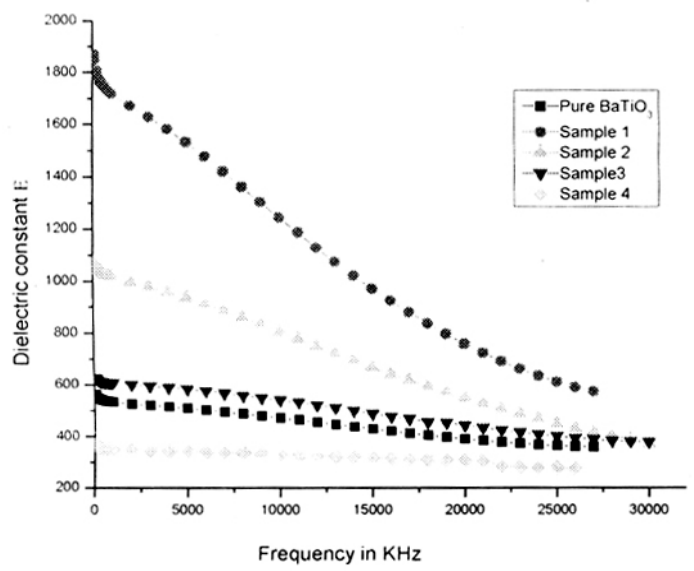

Fig. 2. Frequency vs. Dielectric constant

$\mathrm{BaTiO}_{3}$. The frequency spectra of the conductivity for all the samples show some dispersion with the increase of frequency, which is the mirror image of dielectric constant. It is observed from Fig. 5, that with the decrease of $\mathrm{CeO}_{2}$ content, tan $\delta$ increase up to a frequency of $1 \mathrm{MHz}$ and then decreases with frequency. The width of the loss peak shown in Fig. 5. signifies the possibility of relaxation time. In our present 


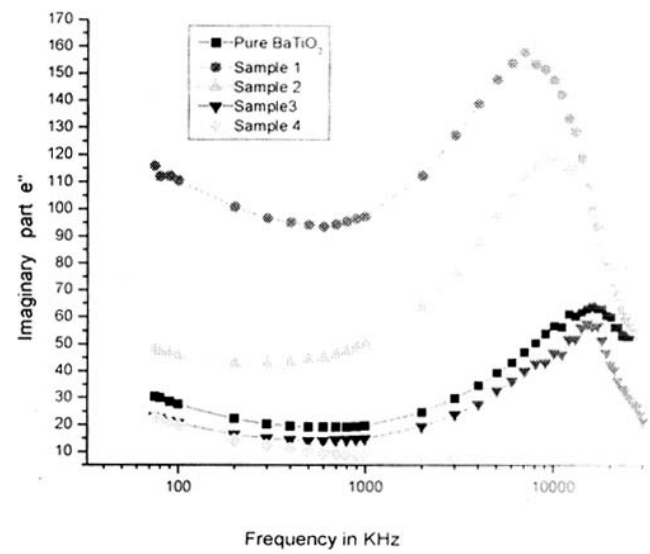

Fig. 3. Frequency vs. imaginary part.

experiment relaxation occurs at $1 \mathrm{MHz}$ for all the samples except for $\mathrm{x}=0.4$. It is evident from Fig. 3. that the imaginary part of dielectric constant $\varepsilon^{\prime \prime}$ increases with the increase of frequency up to a maximum value of about $\sim 1 \mathrm{MHz}$ and then $\varepsilon^{\prime \prime}$ decreases with the further increase of frequency. It is also obvious from this experiment (also shown in Fig. 5) that the dielectric relaxation occurs at about $\sim 1 \mathrm{MHz}$ for all the samples of $\mathrm{Ba}_{1-\mathrm{x}} \mathrm{Ce}_{\mathrm{x}}$ $\mathrm{TiO}_{3}$ for $\mathrm{x}=0.1,0.2$ and 0.3 except for $\mathrm{x}=0.4$. This is to be noted that $\varepsilon^{\prime} . \varepsilon^{\prime \prime}$, $\tan \delta$ and $\sigma_{\mathrm{ac}}$ versus frequency plots show the same behavior pattern but $\varepsilon^{\prime} . \varepsilon^{\prime \prime}$, and $\sigma_{\mathrm{ac}}$ have no frequency dependence of the sample for $\mathrm{x}=0.4$ and differs noticeably from the other concentrations as covered as covered in this experiment. Himanshu et al. ${ }^{(14)}$ observed that the frequency dependant conductivity is independent of temperature. Fig. 2 shows that at lower frequency there is a substantial increase in the dielectric constant which is attributable to a dipolar contribution to $\varepsilon^{\prime}(\omega)$ from the hoping of cations between neighboring atoms.

Table 1

Variation of compositions, $\tan \delta_{\max }, \varepsilon_{\max }, \varepsilon^{\prime \prime}{ }_{\max }$ and lattice parameter ratio of $\mathrm{BaTiO}_{3}$ with Ce concentrations

\begin{tabular}{|c|l|l|l|l|l|}
\hline Sample No. & \multicolumn{1}{|c|}{$\mathrm{Ba}_{1-\mathrm{x}} \mathrm{CexTiO}_{3}$} & \multicolumn{1}{c|}{$\varepsilon_{\max }$} & \multicolumn{1}{c|}{$\tan \delta_{\max }$} & \multicolumn{1}{c|}{$\varepsilon_{\text {max }}^{\prime \prime}$} & \multicolumn{1}{c|}{ c/a } \\
\hline Pure $\mathrm{BaTiO}_{3}$ & $\mathrm{x}=0.0$ & 560.5392 & 0.1557 & 64.01 & 1.008 \\
\hline 1 & $\mathrm{x}=0.1$ & 1868.5392 & 0.1990 & 157.97 & 1.0014 \\
\hline 2 & $\mathrm{x}=0.2$ & 1073.6316 & 0.1598 & 119025 & 1.0017 \\
\hline 3 & $\mathrm{x}=0.3$ & 626.47680 & 0.1211 & 57.60 & 1.00207 \\
\hline 4 & $\mathrm{x}=0.4$ & 396.6850 & 0.0484 & 14.48 & 1.0127 \\
\hline
\end{tabular}

In Table 1 it is shown that adding of small amount of $\mathrm{CeO}_{2}$ increases dielectric constant as well as imaginary part $\varepsilon^{\prime \prime}{ }_{\max }$. The value of dielectric constant increases as $\mathrm{CeO}_{2}$ concentration increases up to $\mathrm{x}=0.1$ and then decreases. Similar results are obtained in the case of dielectric constant vs. temperature characteristics ${ }^{(17)}$. The value of imaginary part $\varepsilon^{\prime \prime}{ }_{\text {max }}$ increases as the Ce concentration goes up to $\mathrm{x}=0.1$ and then decreases. For the sample $\mathrm{x}=0.4$, the value of $\mathrm{c} / \mathrm{a}$ ratio is more than unity. 


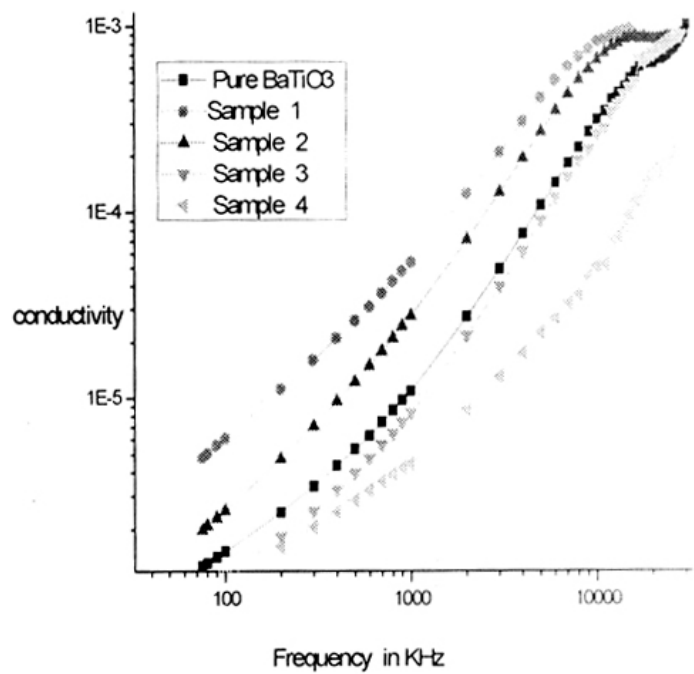

Fig. 4. Frequency Vs. Conductivity

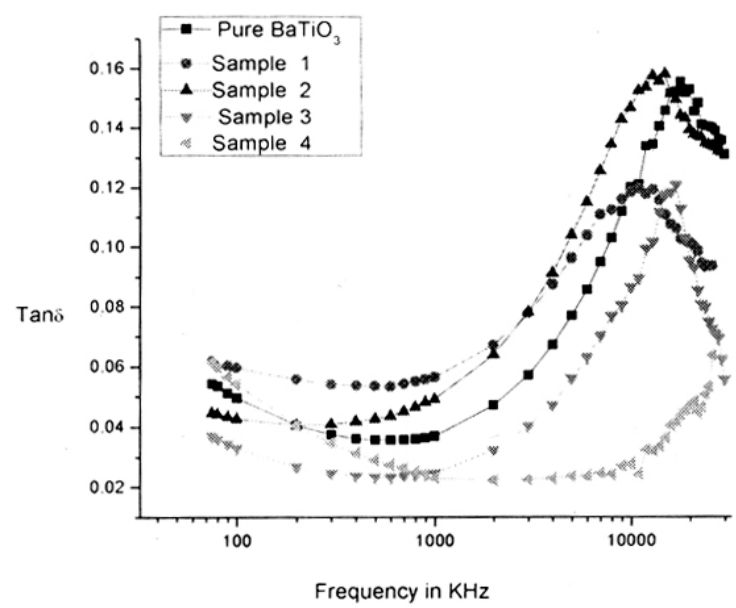

Fig. 5. Frequency Vs. Tan $\delta$.

\section{CONCLUSIONS}

$\mathrm{X}$-Ray defractogram of $\mathrm{Ba}_{\mathrm{x}} \mathrm{Ce}_{1-\mathrm{x}} \mathrm{TiO}_{3}$ synthesized by the solid state reaction shows tetragonal barium titanate. As the concentration of $\mathrm{CeO}_{2}$ increases, the structure changes to hexagonal. This justifies the value of $\mathrm{c} / \mathrm{a}$ ratio to be more than unity for the sample $\mathrm{x}=$ 0.4. Dielectric spectroscopy is a powerful technique for characterizing the doping influence. Analyses of frequency dependence of real and imaginary parts of dielectric permittivity and conductivity, performed at room temperature, show the nature of relaxation. All results suggest that the relaxation phenomena observed in $\mathrm{Ba}_{1-\mathrm{x}} \mathrm{Ce}_{\mathrm{x}} \mathrm{TiO}_{3}$ system can be attributed to the damping of dipole oscillators due to application of external field. 


\section{ACKNOWLEDGEMENTS}

The authors are thankful to Mr. Moinul Ahsan, P.S.O IGCRT (Institute of Glass and Ceramic Testing), BCSIR for giving XRD spectra and Dr. Dilip Kumer Shaha, Material Science Division, BAEC (Bangladesh Atomic Energy Commission) for helping in XRD spectrum analysis. The authors are also thankful to the Director, BCSIR Laboratories. Dhaka, for giving the permission to carry out the research work.

\section{REFERENCES}

5. H. KniepKamp and W. Heywang, "Depolarization Effects in Polycrystalline $\mathrm{BaTiO}_{3} Z$. Angrew. Phys., 6(9), 385-90, 1954.

6. R. C. De. VRies And J. E. Burke, “Microstructure of $\mathrm{BaTiO}_{3}$ Ceramics”, J. Am. Ceram. Soc., 40(6), 200-206, 1957.

7. K. Kinoshita And YamajI, "Grain size effects on dielectric properties in $\mathrm{BaTiO}_{3}$ ceramics," J. Appl. Phys., 47(1), 371-73, 1976.

8. K. S. MAZdyAsni AND L. M. BROWN, "Microstructure and Electrical properties of $\mathrm{Sc}_{2} \mathrm{O}_{3}$ Doped, Rare-Earthed oxide-Doped and undoped $\mathrm{BaTiO}_{3}$. ." J. Am. Ceram. Soc., 54(11), 53943, 1971.

9. D. Kolar, J. P. GuHA And M. Buh, "Ceramic and Dielectric properties of Selected Composition in the $\mathrm{BaO}-\mathrm{TiO}_{2}-\mathrm{CeO}_{2}$ system,” Proc. Br. Ceram. soc., 23 152-58, 1972.

10. R. S. Roth, T. Negas, H. S. Parker, D. B. Minor and C. Jones, "Crystal Chemistry of Cerium Titanates, Tantalates and Niobates,” Mater. Res. Bull., 12, 1173-82, 1977.

11. G. I. TAKura, T. Iguchi, AND T. Kuroda. "Ceramic Dielectric Materials with High Dielectric constant for multilayer Ceramic Capacitors,” Natl. Tech. Rep. (Jpn.), 31(3), 145-56, 1985.

12. H. Hwang And Y. H. Han, “Dielectric properties of $\left(\mathrm{Ba}_{1-\mathrm{x}} \mathrm{Ce}_{\mathrm{x}}\right) \mathrm{TiO}_{3}$.” Jpn. J. Appl. Phys., 39, 701-704, 2000.

13. D. Hennings, B. Schreinemacher and H. Schreinemacher, "High-Permitivity Dielectric Ceramics with high Endurance,” J. Eur. Ceram. Soc., 13, 81-88, 1994.

14. D. Makvoee, Z. SamardziJa And D. Kolar, “Solid Solubility of cerium in $\mathrm{BaTiO}_{3}$ ” J. Solid State Chem., 123, 30, 30-38, 1996.

15. D. Makvoee and D. Kolar, "Internal Oxidation of $\mathrm{Ce}^{3+}-\mathrm{BaTiO}_{3}$ Solid Solutions,” J. Am. Ceram. Soc., 80(1), 15-52, 1997.

16. Y. PARK AND H. G. KIM, "Dielectric Temperature Characteristics of Cerium Modified Barium Titanate based ceramics with core shell Grain Structure,” J. Am. Ceram. Soc., 80(1), 106-12, 1997.

17. A. Chen, Y. Zhi, J. Zhi, P. M. Vilarionho AND J. I. Baptista, "Synthesis and characterization of $\mathrm{Ba}\left(\mathrm{Ti}_{1-\mathrm{x}} \mathrm{Cex}\right) \mathrm{O}_{3}$ ceramics,” J. Eur. Ceram. Soc., 17, 2117-21, 1997.

18. A. K. Himanshu, D. G. Gupta And T. P. Sinha, "Dielectric Study of $\mathrm{Ba}\left(\mathrm{Zn}_{1 / 3} \mathrm{Nb}_{2 / 3}\right) \mathrm{O}_{3}$ at low temperature,” Ind. J. of Pure and App. Phys., 44. 391-397, May 2006.

19. W. WERSING, "Electronics ceramics", Elseiver, London,” 1991. 
20. N. M. Molokhia, M. A. A. Issa And S. A. Nasser, J. Am. Ceram. Soc., 67, 289-291, 1984.

21. Jin Hyun Hwang And Young Ho Han, "Electrical Properties of Cerium - Doped $\mathrm{BaTiO}_{3}$," J. Am. Ceram. Soc., 84(8), 1750-1754, 2001.

Journal of Bangladesh Academy of Sciences, Vol. 32, No. 2, 79-85, 2008 